\title{
Molecular cytogenetic differentiation of paralogs of Hox paralogs in duplicated and re-diploidized genome of the North American paddlefish (Polyodon spathula)
}

Radka Symonová ${ }^{1,2^{*}}$, Miloš Havelka ${ }^{3}$, Chris T. Amemiya ${ }^{4}$, William Mike Howell ${ }^{5}$, Tereza Koř́nková1, Martin Flajšhans ${ }^{3}$, David Gela ${ }^{3}$ and Petr Ráb ${ }^{1}$

\begin{abstract}
Background: Acipenseriformes is a basal lineage of ray-finned fishes and comprise 27 extant species of sturgeons and paddlefishes. They are characterized by several specific genomic features as broad ploidy variation, high chromosome numbers, presence of numerous microchromosomes and propensity to interspecific hybridization. The presumed palaeotetraploidy of the American paddlefish was recently validated by molecular phylogeny and Hox genes analyses. A whole genome duplication in the paddlefish lineage was estimated at approximately 42 Mya and was found to be independent from several genome duplications evidenced in its sister lineage, i.e. sturgeons. We tested the ploidy status of available chromosomal markers after the expected rediploidization. Further we tested, whether paralogs of Hox gene clusters originated from this paddlefish specific genome duplication are cytogenetically distinguishable.

Results: We found that both paralogs HoxA alpha and beta were distinguishable without any overlapping of the hybridization signal - each on one pair of large metacentric chromosomes. Of the HoxD, only the beta paralog was unequivocally identified, whereas the alpha paralog did not work and yielded only an inconclusive diffuse signal. Chromosomal markers on three diverse ploidy levels reflecting different stages of rediploidization were identified: quadruplets retaining their ancestral tetraploid condition, semi-quadruplets still reflecting the ancestral tetraploidy with clear signs of advanced rediploidization, doublets were diploidized with ancestral tetraploidy already blurred. Also some of the available microsatellite data exhibited diploid allelic band patterns at their loci whereas another locus showed more than two alleles.

Conclusions: Our exhaustive staining of paddlefish chromosomes combined with cytogenetic mapping of ribosomal genes and Hox paralogs and with microsatellite data, brings a closer look at results of the process of rediploidization in the course of paddlefish genome evolution. We show a partial rediploidization represented by a complex mosaic structure comparable with segmental paleotetraploidy revealed in sturgeons (Acipenseridae). Sturgeons and paddlefishes with their high propensity for whole genome duplication thus offer suitable animal model systems to further explore evolutionary processes that were shaping the early evolution of all vertebrates.
\end{abstract}

Keywords: HoxA/D paralogs mapping, Sturgeon whole genome duplication, Ancient fish genome, Rediploidization

\footnotetext{
* Correspondence: radka.symonova@uibk.ac.at

'Laboratory of Fish Genetics, Institute of Animal Physiology and Genetics,

Czech Academy of Sciences, 277 21, Liběchov, Czech Republic

${ }^{2}$ Research Institute for Limnology, University of Innsbruck, Mondseestr. 9,

Mondsee, Austria

Full list of author information is available at the end of the article
} 


\section{Background}

The North American (Polyodon spathula) and Chinese paddlefish (Psephurus gladius), i.e. fishes with paddlelike snout, are the only extant representatives of an early radiation of ray-finned fishes recognized as the family Polyodontidae within the order Acipenseriformes [1]. This ancient lineage, i.e. sturgeons, shovelnoses (Acipenseridae) and paddlefishes, represents an archaic group known to be at least as old as the early Jurassic (some 200-175 Mya). Polyodontids and acipenserids diverged from one-another in the Jurassic period 180-140 Mya [2]. Paddlefishes form a distinct monophyletic clade within Acipenseriformes as evidenced by molecular phylogeny [3].

There is a consistent body of evidence on the chromosome number of the North American paddlefish but discordant data on its genome size, yet all authors consider this species palaeotetraploid. Namely, Dingerkus and Howell [4] based on karyotyping of 31 cells from two paddlefish males found the $2 \mathrm{n}=120$ and reported a karyotype apparently composed of twelve quadruplets of macro- and 72 microchromosomes and thus hypothesized a tetraploid origin in this species. Later, the $2 n=120$ was reconfirmed and nuclear DNA content corresponding to genomes of sturgeons with $2 n=120$ was reported by Zhang et al. [5]. Birstein et al. [6] re-examined nuclear DNA content of this species and confirmed its palaeotetraploid status. The Chinese paddlefish (Psephurus gladius) has $2 \mathrm{n}=120$ and nuclear DNA content corresponding to sturgeon genomes with $2 \mathrm{n}=120$ was reported [5]. All available data indicate a palaeotetraploid origin of the paddlefish lineage, similarly as in a number of sturgeon species [7].

The cytogenetic analyses of sturgeons and paddlefishes is challenging since, in addition to macrochromosomes, extremely small-sized microchromosomes typically constitute a substantial proportion of the acipenseriform karyotype and their morphology has long been characterized as indistinguishable [8]. However, a recent work [9] reports morphology of these small-sized chromosomes that would have been called "microchromosomes". Further details on sturgeon genetics and cytogenetics related to ploidy level were summarized by Havelka et al. [10] and Trifonov et al. [11].

Sturgeons generally exhibit a remarkable propensity for hybridization and polyploidization resulting in viable and even fertile highly polyploid individuals and interspecific (allopolyploid) hybrids [12-15]. Each such whole genome duplication (WGD) is followed by a rediploidization [16]. During this process, a partial retention of $4 n$ features is observable, like chromosome numbers and their external morphology (e.g. [17]). Distinct signs of ongoing and to different extent advanced rediploidization can be found in other cytogenomic markers - e.g.
HoxA/D sequences in paddlefish [12] and microsatellite studies in sturgeon $[18,19]$.

Recently, molecular analyses of paralogs of both HoxA and HoxD gene clusters demonstrated a specific WGD event in the paddlefish lineage dated about 42 million years ago (Mya) (for more details on dating [12]) and independent from the multiple WGDs in sturgeons [19]. Other comparable events among ray-finned fish are the salmonid specific WGD (called SR) dated about 88 Mya [20] and the teleost specific WGD (TSGD) dated about 320 Mya [21]. Paddlefish thus offers a model system complementary to others to study consequences of WGD by molecular cytogenetics in the light of clear indications of secondary rediploidization at the molecular level in HoxA/D genes clusters (e.g. [12]).

Hox genes clusters are expressed along the anteroposterior axis of all bilaterians and play a key role in animal development [22]. In vertebrates, four paralogous Hox clusters (Hox A, B, C, and D) originate from a single Hox cluster of the last common ancestor of vertebrates and cephalochordates ([23], [24] reviewed in [25]). These four paralogs arose by two ancestral consecutive WGD events known as R1 and R2 that occurred in the vertebrate stem lineage around 525 Mya $[24,26]$. The paddlefish specific WGD gave rise to a further order of paralogs called here $\alpha$ and $\beta$, respectively, as introduced by [12].

In this study, using FISH (fluorescence in situ hybridization) we co-hybridized the HoxA $\alpha$ with $\operatorname{HoxA} \beta$ and HoxD $\alpha$ with $\operatorname{HoxD} \beta$ of these paddlefish specific paralogs (BAC DNA produced by Crow et al. [12]) to paddlefish chromosomes. This was combined with FISH with ribosomal (rRNA) genes, with conventional chromosome banding and microsatellite analysis to explore consequences of the paddlefish specific WGD and the subsequent rediploidization. The available markers and analyses, including those based on external chromosomal morphology, enabled us to assess the tetraploidy retention versus the extent of rediploidization on the level of coding regions, repetitive sequences, heterochromatin accumulations supplemented with microsatellite analysis.

\section{Results \\ Karyotype and chromosome analysis}

Karyotype analysis re-confirmed the diploid chromosome number $2 \mathrm{n}=120$ and enabled an arrangement of chromosomes into quadruplets, doublets and semiquadruplets where possible (Fig. 1). This arrangement reflected the external morphological features of chromosomes and also information retrievable from the DAPI (i.e., AT-rich regions)/CMA 3 (Chromomycin A3, GC-rich regions specific) fluorescent staining. Firstly, a $\mathrm{CMA}_{3}^{+}$arm of a pair of small sub metacentric macrochromosomes (Fig. 1b). Secondly, DAPI ${ }^{+}$ 


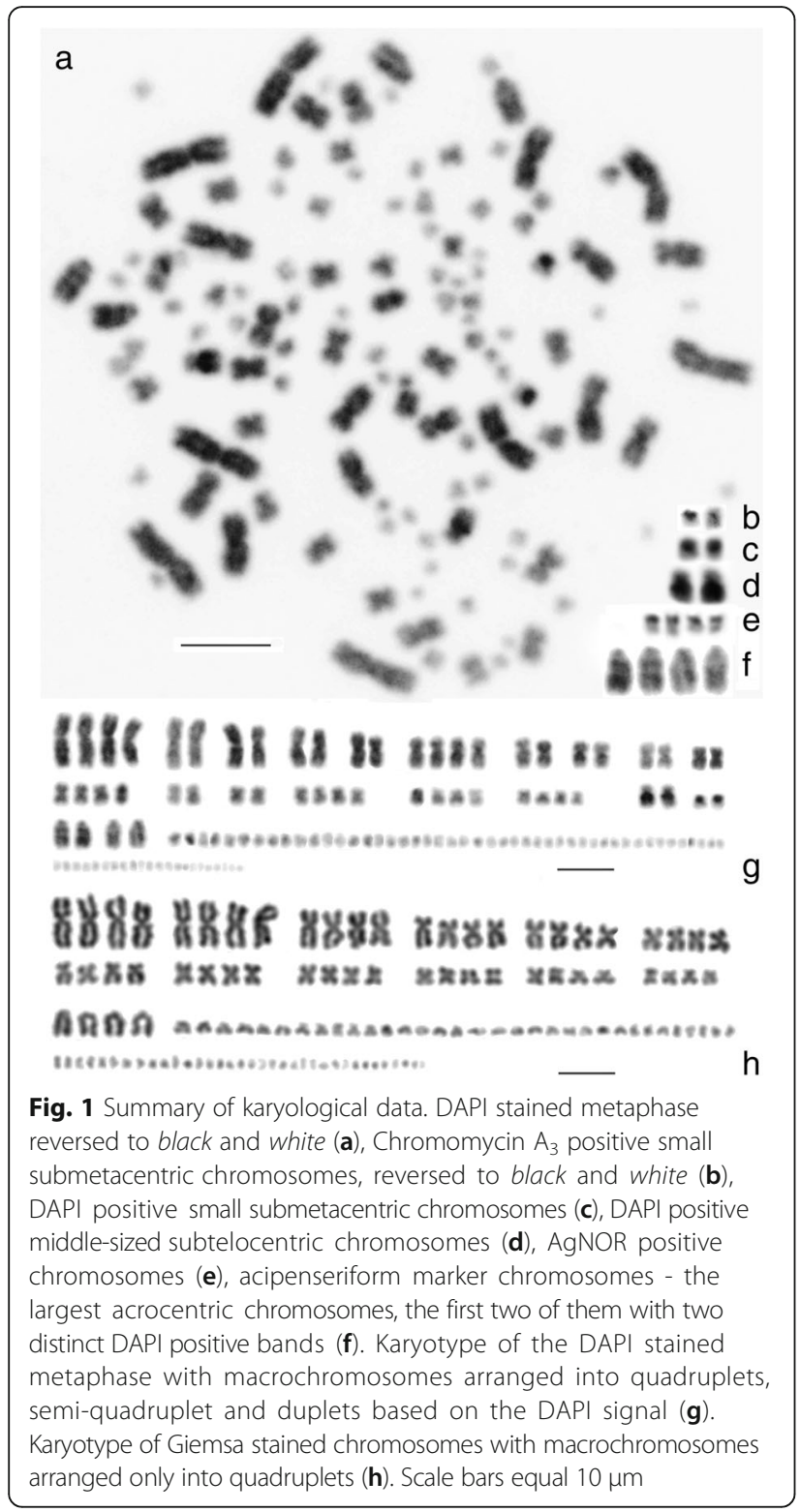

arm of a small submetacentric chromosomes (Fig. 1c). Thirdly, an interstitial $\mathrm{DAPI}^{+}$band on the larger arm of a pair of submetacentric chromosomes (Fig. 1d). DAPI staining demonstrated that there is no other pair of submetacentric chromosomes with comparable external morphology and banding pattern. Further, two pairs of the largest "acipenseriform" acrocentric chromosome markers were distinguishable based on their morphology in the tetraploid condition. However, the DAPI fluorescence revealed AT-rich signals accumulated in form of a double-band in only two of these four chromosomes (Fig. 1f). The $\mathrm{AgNO}_{3}$ staining (visualizing chromosomes with the active ribosomal genes, the AgNORs) consistently yielded a quadruplet of transcriptionally active major ribosomal sites on four small metacentric macrochromosomes (Fig. 1e). The two $\mathrm{CMA}^{3+}$ signals (Fig. 1b) did not co-localize with the active NOR sites (Fig. 1e).

\section{FISH experiments}

The FISH with $28 \mathrm{~S}$ ribosomal DNA (rDNA) showed up to 12 signals on mostly small-sized macrochromosomes or microchromosomes (Fig. 2a). The FISH with 5S rDNA revealed signals on three morphologically different pairs of chromosomes (Fig. 2b): i) small dot-like signals interstitially on one arm of a pair of large metacentric chromosomes; ii) large signals on a whole arm of a pair of small metacentric macrochromosomes, and iii) dot-like signals on a pair of small metacentric macrochromosomes. FISH with telomeric repeats (TTAGGG) $\mathrm{n}$ did not reveal any interstitial telomeric sites (not shown).

FISH with $\operatorname{Hox} A \alpha / \beta$ and $\operatorname{HoxD} \beta$ gene clusters, respectively, showed a clear $2 \mathrm{n}$ pattern of the respective paralogs (Fig. 2c-d). Namely, the HoxA $\alpha$ paralog was physically mapped to the telomeric region of a pair of large metacentric chromosomes (Fig. 2c, red signals), the HoxA $\beta$ paralog mapped also to telomeric region of another pair of large metacentric chromosomes (Fig. 2c, green signals) with no overlapping signals. The $\operatorname{HoxD} \beta$ paralog mapped to the telomeric region of one pair of the largest acrocentric chromosomes (Fig. 2d, red

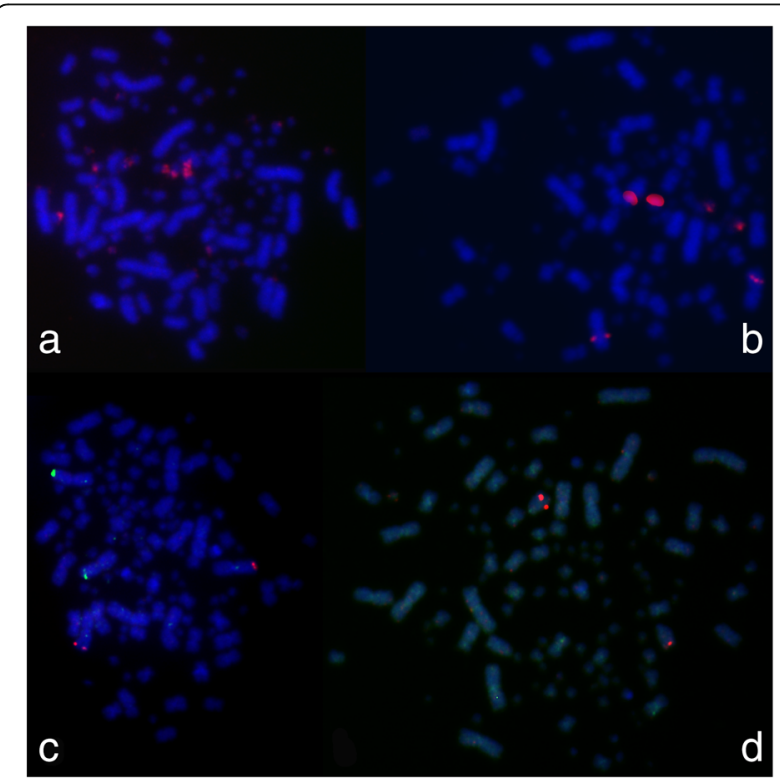

Fig. 2 FISH with rDNAs and Hox clusters. FISH with $28 \mathrm{~S}$ rDNA (a, red), $5 S$ rDNA $(\mathbf{b}$, red) and FISH with BACs of the paralogs Hox-A alpha (c, red) and Hox-A beta (c, green) and of the paralogs Hox-D beta (d, red) and Hox-D alpha (d, green - the diffuse character of this signal causes a greenish coloration of almost all chromosomes). Scale bars equal $10 \mu \mathrm{m}$ 
signals). The HoxD $\alpha$ paralog yielded an inconclusive interspersed FISH signal and it was impossible to localize this region even with using competitor DNA (Fig. 2d, green and diffuse greenish signals on majority of chromosomes).

\section{Integration of karyological and molecular cytogenetic markers}

Integrating all analyzed markers, the following groups of chromosomes (labeled with abbreviations for later reference) were identified to compose the karyotype: M1 quadruplet of the largest metacentric chromosomes of equal size (according to external morphology); M2 semiquadruplet of slightly smaller large metacentric chromosomes based on HoxA paralogs mapping; M3 semiquadruplet of meta- to submetacentric chromosomes of intermediate size including one smaller and one larger doublet respectively based on size and 5S rDNA FISH signals; SM1 semi-quadruplet of submetacentric chromosomes of intermediate size; SM2 semi-quadruplet; SM3 semi-quadruplet; SM4-5 (semi-quadruplets/doublets of small meta- to submetacentric chromosomes, these chromosomes are difficult to classify into separate groups and pair with the exception of the AgNOR quadruplet; ST1 semi-quadruplet/doublet of a conspicuous pair of submeta- to subtelocentric chromosomes with a remarkable $\mathrm{DAPI}^{+}$band on the larger arm. This pair of chromosomes lacks any counterpart in its size and morphological category, however, it can be linked with a pair of small metacentric chromosomes with a distinct DAPI band on one arm; A1 semi-quadruplet of medium-size acrocentric chromosomes (the acipenseriform acrocentric markers). There is a $\mathrm{DAPI}^{+}$double-band on two of these chromosomes and the $\operatorname{HoxD} \beta$ paralog FISH signal on the other two chromosomes; $\mathrm{m} 1$ doublet of very small chromosomes (microchromosomes) with a clear $\mathrm{CMA}_{3}^{+}$band; $\mathrm{m} 2$ doublet of very small chromosomes (microchromosomes) with a clear $\mathrm{DAPI}^{+}$band. In this way, we identified 54 macrochromosomes (or microchromosomes with features enabling their identification) and 66 microchromosomes (i. e. small chromosomes without any cytogenetic features).

Contrary to these observations yielded by fluorescent stainings, AgNOR staining and C-banding, the Giemsa staining produced only rough and uniform external chromosomal morphology corresponding approximately to tetraploid condition. There were no apparent characteristics enabling any possibility to distinguish the aforementioned quadruplets, semi-quadruplets and duplets (Fig. 1h).

All cytogenetic data are visualized and summarized in the Fig. 3.

To sum up, the ancestrally palaeotetraploid paddlefish chromosomal complement $(2 \mathrm{n}=120)$ represents a complex mosaic structure consisting of characters with $1 /$ retained tetraploidy (AgNORs); 2/partially retained tetraploidy, i.e. apparent ancestral tetraploidy with clear signs of secondary diploidization (acipenseriform acrocentric marker, HoxA and HoxD loci); 3/diploidized characters $\left(\mathrm{DAPI}^{+}\right.$and $\mathrm{CMA}_{3}^{+}$heterochromatic sites); and 4/characters with apparent chromosomal re-arrangements (5S rDNA sites). The situation in $5 \mathrm{~S}$ rDNA sites did not match neither to ancestral tetraploidy nor to the diploidized condition.

Quantification of repetitive sequences in BAC clones used as FISH probes

Proportion of repeats in the BAC DNA used as FISH probes can be potentially crucial for functionality of

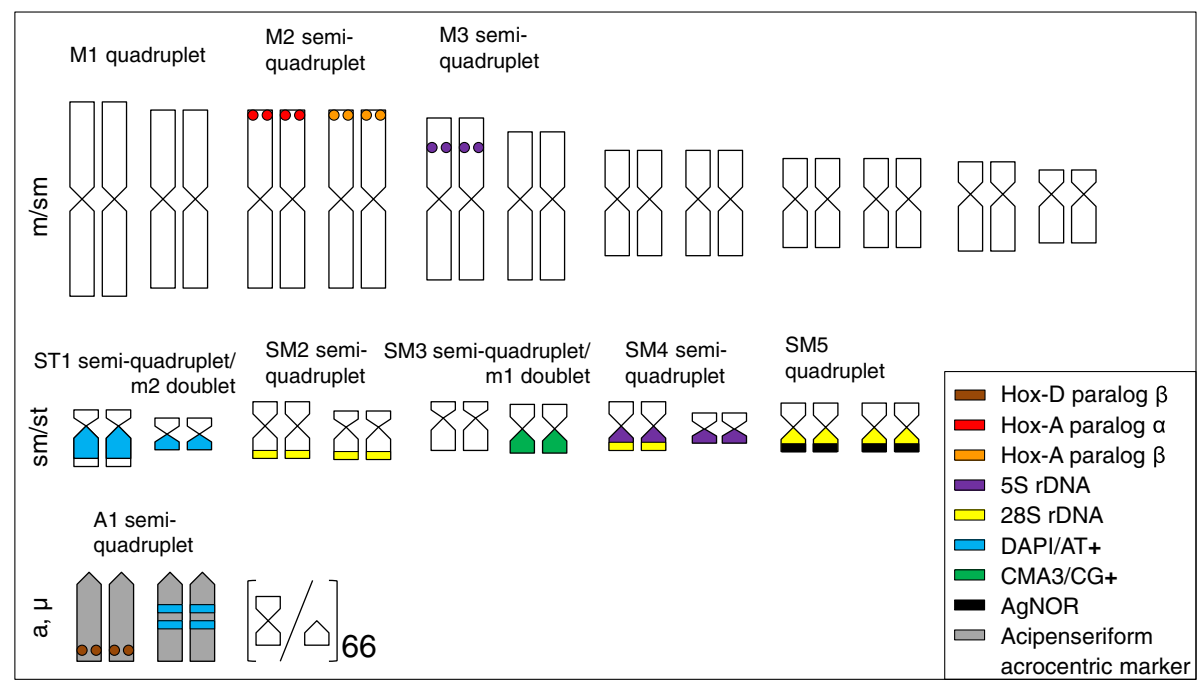

Fig. 3 Ideogram summarizing all chromosomal markers investigated in this study 
FISH experiments since repeats interspersed throughout genome can yield signals unspecific for the BAC DNA under study. In Table 1, we summarize repeats proportion for each BAC DNA.

\section{Microsatellite genotyping}

From all together eleven tested sturgeon microsatellite markers, only the locus Afu 68 [27] displayed consistent amplification in P. spathula. This locus exhibited diploid allelic band pattern across all analyzed samples. Similarly, P. spathula specific loci (see Additional file 1, Supplementary Methods) had diploid allelic band patterns. An exception to this general pattern was shown at the locus Psp-29 [28] for which more than two alleles were observed (Fig. 4; Additional file 2, Supplementary Results).

\section{Discussion}

This study demonstrated an in situ localization of three of four available paralogs of the HoxA and HoxD gene clusters in P. spathula. Both HoxA paralogs were clearly distinguishable without any FISH signal overlaps showing that the sequence divergence was high enough to enable their unequivocal discrimination under high stringency conditions. The sequence divergence between the full coding regions varied among gene clusters ranging from 2.12\% (Hox-A11) to 10.94\% (Hox-D13) [12]. The proportion of repetitive sequences was identified to be approximately $10 \%$ where ascertainable (Table 1 ). The repetitive sequences, if specific for the Hox clusters, might have contributed to discrimination of Hox paralogs. On the other hand, the failure in localization of the HoxD $\alpha$ can also be ascribed to accumulation of repetitive sequences that were spread also throughout the rest of genome and thus prevented from an accurate localizing of this paralog. This result contributes as a comparative reference to the future attempts to localize paralogous regions and presents an example of linking genomic approach with molecular cytogenetics. Further, our results made it possible to better assess genome evolution after the paddlefish specific WGD on the finer-scale level in combination with other cytogenetic markers and to compare it with the outer chromosomal morphology based on conventional cytogenetics as shown by [4].
Ray-finned fishes provide an outstanding model to investigate WGDs and their consequences because of their complex evolutionary history involving among vertebrates unprecedented propensity for hybridization and polyploidization and their genome plasticity tolerating high variability in genome size and chromosome number [29].

Two basic ways of chromosomal evolution following a WGD event were documented among ray-finned fishes: $1 /$ conserving of the chromosome numbers after WGD which exists in more than $50 \%$ of teleosts after the TSGD [30], in polyploid cyprinid lineages [31] and in most acipenseriforms [19], and 2/extensive chromosomal re-arrangements leading to diversified chromosome numbers, e.g. in salmonids (reviewed by [32]). Other lineages known to have experienced WGD [33] need to be cytogenetically documented.

Acipenserids, the sister lineage of polyodontids, underwent at least three rounds of lineage specific WGDs $[19,34,35]$. The first one occurred in their common (already extinct) ancestor with $2 \mathrm{n}=60$ leading to a $\sim 120$-chromosomes lineage with presently unknown dating. The second lineage specific WGD took place separately in the Atlantic sturgeon lineage ( $53 \mathrm{Mya})$ and in the Pacific lineage ( $70 \mathrm{Mya})$. The third WGD is unique to the Shortnose sturgeon (Acipenser brevirostrum) dated 235 Mya [2]. Polyodon spathula inhabits the same range as some species of the Atlantic sturgeon lineage. The $P$. spathula specific WGD is supposed to have occurred $\sim 42$ Mya [12] which is a timing somewhat similar to the WGD of the Atlantic sturgeon lineage [2]. Acipenserids split from polyodontids $\sim 170 \mathrm{Mya}$ and the divergence time between the Pacific and the Atlantic lineage appears as about 121 Mya [2]. Assuming WGD specific to $P$. spathula [12] and no presence of WGD specific to Atlantic lineage species with 120 chromosomes [2], the first specific WGD in Acipenseridae had to take place between split of polyodontids from acipenserids ( 170 Mya) and split of Atlantic and Pacific lineage of Acipenseridae ( 121 Mya; Fig. 5).

Chromosome numbers and their outer morphology in both acipenserids and polyodontids remain mostly conserved in the post-WGD situation [4]. Regarding the longevity estimated in the paddlefish WGD, this conservatism might be explained by two possible factors and/ or by combination thereof: $1 /$ significantly reduced rate

Table 1 Overview of BACs of Hox paralogs used as FISH probes

\begin{tabular}{llllrr}
\hline Clone ID sensu [12] & BAC & Paralogs & GenBank Accession Nr. & Size (bp) & Repeats (\%) \\
\hline BAC231C24 & 1816 & Hox-D 8-13a & JX280946.1 & 22.134 & 33.595 \\
BAC249G23 & 1817 & Hox-D 8-13ß & JX280945.1 & 131.867 & 9.17 \\
BAC352P4 & 1818 & Hox-A 1-13a & JX448769.1 & 139.159 & 10.66 \\
BAC370N10 & 1819 & Hox-A 1-13ß & JX448770.1 & 10.48 \\
\hline
\end{tabular}




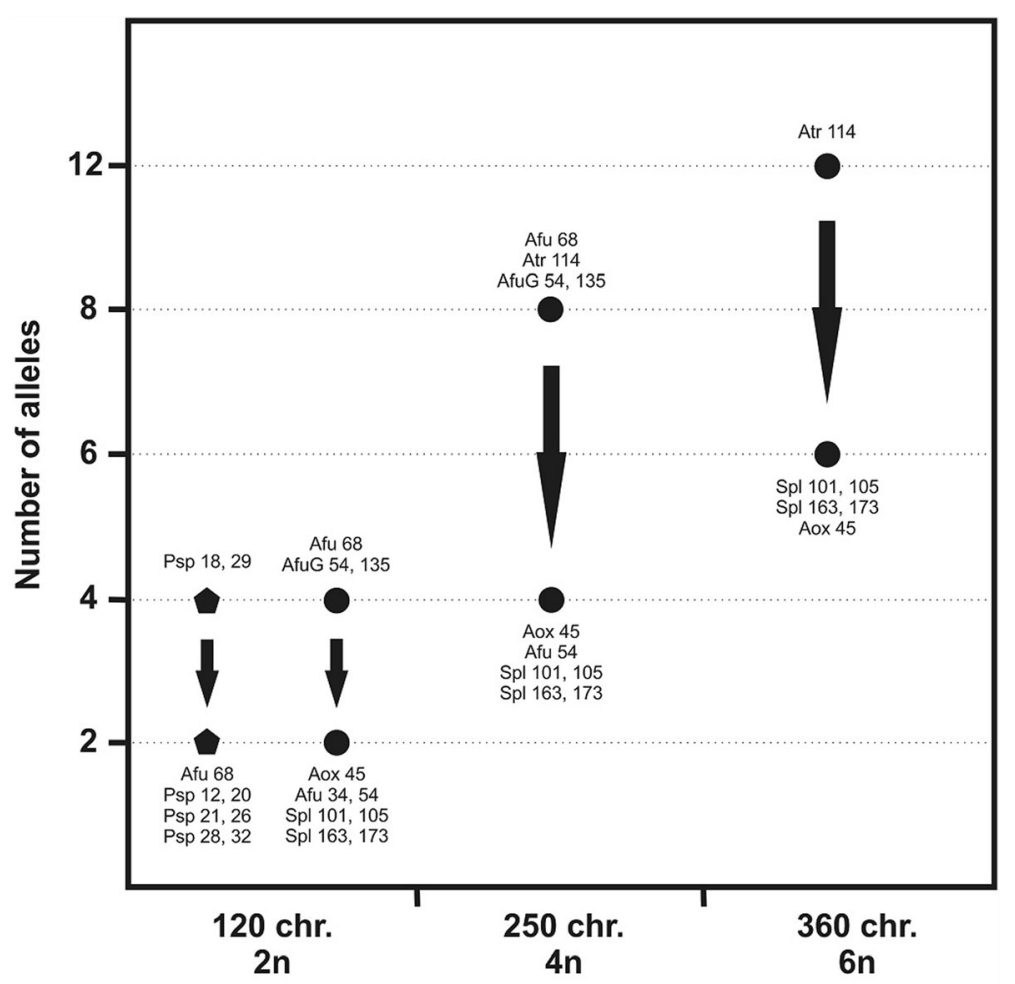

Number of chromosomes and ploidy levels

Fig. 4 Microsatellite analysis of ploidy level. Microsatellite data (number of alleles) in Polyodon spathula and comparison with sturgeons related to chromosomes numbers and ploidy levels provide evidence of partial genome rediploidization in P. spathula ( $)$ and sturgeons $(\bullet)$. The figure is based on data from this study and previous studies $[18,28]$

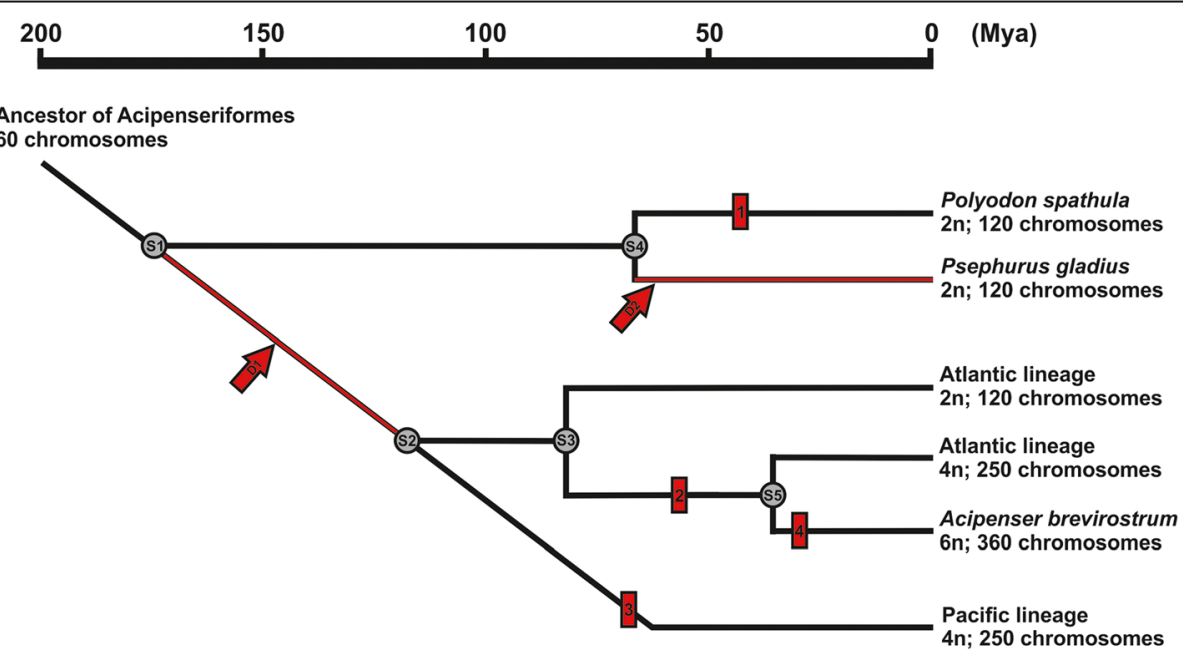

Fig. 5 Suggested main events in evolution of Acipenseriformes. S1 = split of Polyodontidae and Acipenseridae ( 170 Mya); S2 = Split of Atlantic and Pacific lineage in Acipenseridae ( 121 Mya); S3 = split of 2n and 4n species within Atlantic lineage ( 80 Mya); S4= Split of Polyodon spathula and Psephurus gladius ( 68Mya); S5 = Split of Acipenser brevirostrum ( 36 Mya). Whole genome duplication (WGD) events are: $1=$ WGD specific to Polyodon spathula $(60 \rightarrow 120$ chromosomes; 42 Mya [12]); $2=$ WGD in Atlantic lineage $(120 \rightarrow 250$ chromosomes; 53 Mya); $3=$ WGD in Pacific lineage $(120 \rightarrow 250$ chromosomes; 70 Mya); $4=$ WGD specific to Acipenser brevirostrum $(250 \rightarrow 360$ chromosomes 35 Mya); D1 = the first WGD in Acipenseridae $(60 \rightarrow 120$ chromosomes) had to take place between $\sim 170$ Mya and $\sim 121$ Mya; D2 = probable WGD (60 $\rightarrow 120$ chromosomes) specific to Psephurus gladius. The data are based on study by Peng et al. [2] and Crow et al. [12] 
of molecular evolution (both mitochondrial and nuclear) that was reported in both coding and non-coding sequences as well as in chromosomal evolution [36, 37]. It can be assumed that also the rate of secondary diploidization in paddlefish and acipenserids might have been influenced by this generally reduced rate of evolution; $2 /$ morphology of chromosomes undergoing WGD should be taken into account. Once exclusively or mostly metacentric chromosomes undergo a WGD (as in acipenseriforms and also in cyprinids), there is a narrower spectrum of potential chromosome rearrangements to be employed within the duplicated sets of chromosomes. This factor might have contributed to the situation shown here on paddlefish chromosomes. Whereas e.g. in salmonids, chromosomes of the hypothetical $2 \mathrm{n}$ ancestor might have been composed of mostly acrocentric chromosomes [38] which facilitates chromosomal evolution via Robertsonian fusions. Such mechanism was recently described in human acrocentrics harboring rDNA - under certain circumstances acrocentrics physically link via their centromeres and become fusogenic [39]. From our previous studies we know that salmonids possess amplified to extremely amplified rDNA sites mostly in centromeres of acrocentric chromosomes [40, 41]. These might have been the factors contributing to the numerous centric fusions described in all salmonid lineages [32] and at the same time contributing to the conservatism preserving the post-WGD chromosome number in the paddlefish. However, more detailed analyses on the finer scale as shown here by molecular cytogenetic approach or by sequencing of Hox genes paralogs [12] revealed advanced stages of diploidization in coding (HoxA/D gene clusters) and some non-coding regions (accumulation of $\mathrm{AT}^{+}$- and $\mathrm{GC}^{+}$-rich heterochromatic regions; microsatellites). This indicates dynamic processes on multiple levels in the acipenseriform lineage despite the chromosomal morphology prevented from Robertsonian fusions. On the other hand, as evidenced by the persisting of tetraploid state in AgNORs and four active nucleoli per nucleolus, it is advantageous to retain the elevated structural and functional ploidy level in rRNA genes. The multiplied number of non-active 28S rDNA sites on mostly small chromosomes suggests tendencies to spread these regions across chromosomes as evidence in other fishes (e.g. [41, 42]). Our findings of a complex mosaic of diploid, tetraploid and intermediary chromosomal features are in line with the concept of segmental paleotetraploidy revealed recently in the sterlet (Acipenser ruthenus) by chromosome painting [9].

In paddlefish, the re-diploidization process on the karyotype level might have occurred without a change of chromosome counts but by finer-scale re-arrangements of some chromosomes. The fact that some chromosomes and markers still retain the quadruplet nature (i.e. ancestral $4 n=120$ ), supports the hypothesis that the diploid ancestor before the WGD event possessed $2 n=60$ as proposed for the whole group of Acipenseriformes [4, 7].

For further comparisons of consequences of WGDs, more recent entirely polyploid lineages and families within Cypriniformes [33] as e.g. Cyprinus carpio (about $12 \mathrm{Mya}$; David et al. 2003) are available among ray-finned fish. This would be one of the most recent genome duplications among vertebrates indicating a higher incidence of WGD in fishes than in other vertebrate groups [43]. These authors report partially duplicated genome structures and disomic inheritance despite clearly tetraploid chromosome number $(2 \mathrm{n}=100)$ in $C$. carpio demonstrating the complexity of genome evolution in this group. There are also reports on recurrent allopolyploidization events within the Carassius complex [44] proving thus the suitability of ray-finned fish that provide almost a continuum in WGD events on the time scale. However, there are no comparable results available yet to assess the process of rediploidization.

\section{Origin of the acipenseriform acrocentric chromosome marker and its relation to the ploidy level}

One of the four Hox clusters - the HoxD $\beta$ paralog mapped to two of the four largest acrocentric chromosomes while both of the HoxA $\alpha / \beta$ paralogs to four large metacentric chromosomes (the M2 group). Therefore we assume that the largest acrocentric chromosomes originated from one of the large metacentric chromosomes that also represent the ancestral location of Hox clusters. These acipenseriform acrocentric marker chromosomes are known to reflect the ploidy level and used to ploidy level estimation (sensu [13]). They occur in all sturgeons (details on the online database, http://sveb.unife.it/it/ ricerca-1/laboratori/geneweb). Presumably, the Hox clusters were residing on one pair of chromosomes before the $1 R$, i.e. the first vertebrate WGD (as shown by [45] in Amphioxus) and on two pairs before the 2R (the second vertebrate WGD). Hence, there were four pairs of Hox bearing chromosomes before the paddlefish specific WGD, which resulted in eight pairs (i.e. HoxA-D/ $\alpha-\beta$; here, we have investigated four of them and localized three of them). The largest acrocentric chromosomes might thus have arisen by fission of one pair of the metacentric chromosomes. This is in line with the assumption that the numerous metacentric chromosomes represent the ancestral chromosomal morphology. The few acrocentric chromosomes may represent rather exceptional derivatives of metacentrics of more recent origin. This scenario is supported by the fact that no interstitial telomeric sites were found in paddlefish and therefore nothing suggests the opposite way of origin of metacentric chromosomes by centric fusions as proposed by Birstein [46]. This 
scenario finds its support in Acipenser ruthenus, where a single acrocentric pair shares FISH signals of a painting probe derived from the seventh pair of the large metacentrics [9]. This suggests that the fission of the ancestral pair of metacentric chromosomes might have happened already before the split of polyodontids and acipenserids or repeatedly in both lineages. These authors explicitly deny the presence of the second pair of the largest acrocentrics in individuals they investigated [9]. On the other hand, the other two largest acrocentric chromosome are clearly observable in A. ruthenus as published by other authors (see the above mentioned online database maintained by F. Fontana). Hence, there are apparent lineage specific trends and differences in the chromosomal evolution between the polyodontids and acipenserids.

\section{Microsatellite analyses are in line with cytogenetic findings}

Microsatellite data in Acipenseriformes were recently presented by Havelka et al. [18]. They identified sturgeon species in palaeotetraploid condition that were functionally diploid showing a diploid allelic band pattern in some microsatellite loci whereas residual tetraploid pattern in other ones. They further identified functionally tetraploid palaeooctaploids showing tetraploid patterns in some loci and residual octaploid patterns in other ones. Finally, a special situation was described in the functionally hexaploid palaeododecaploid $A$. brevirostrum which also showed hexaploid vs. residual dodecaploid patterns (for details [18]). Such observation of coexistence of diploid and tetraploid or tetraploid and octaploid allelic band patterns in one genome of sturgeon species might reflect functional rediploidization as an ongoing process in this fish lineage (e.g. [19]). This process is expected in polyploids until their complete rediploidization [16]. However, even in fully diploidized genomes, residual evidence for polyploid ancestry (e.g. residual polysomy) is occasionally observed (e.g. in salmonids, [47]). Since paleotetraploid acipenseriform species were considered to be basal group of Acipenseriformes [3], the process of rediploidization probably reaches further than in paleooctaploid species [19]. In light of all these facts, the observation of the duplicated locus Psp-29 in this study and coexistence of diploid and tetraploid allelic band patterns reported by Heist et al. [28] at several microsatellite loci of $P$. spathula supported our observation of partial rediploidization in P. spathula genome from the molecular point of view.

The estimation of locus ploidy by microsatellite genotyping may suffer from inbreeding of analyzed individuals. This might be the case of our microsatellite data as all analyzed individuals originated from a pet shop and we could not exclude their relatedness. Except the locus Psp 18, the estimated ploidy of analyzed loci was in accordance with Heist et al. [28] providing confidence for our conclusion based on microsatellite data. Heist et al. [28] suggested tetraploidy for the locus Psp 18, while we did not observe more than two alleles at the locus (Additional file 2, Supplementary Results). This inconstancy may be caused by close relatedness of analyzed individuals in our study.

Our study presented here and the recently published work [9] performed in sterlet (Acipenser ruthenus) represent the first steps towards a better understanding of processes involved in the rediploidization after WGD events in Acipenseriformes. Both works, although utilizing slightly different approaches of molecular cytogenetics, intersect in presenting a complex mosaic structure consisting of $2 \mathrm{n}$ and $4 \mathrm{n}$ chromosomes segments referred to as "segmental paleotetraploidy" by [9]. Both works thus show the complexity and at the same time the importance of this issue.

\section{Conclusions}

We have shown that the paddlefish Polyodon spathula, in which the chromosome numbers and chromosome morphology remain mostly conserved in the post-WGD situation, do nevertheless show signs of ongoing rediploidization. By combining karyological and molecular cytogenetic markers we were able to distinguish three diverse ploidy levels: tetraploidy (AgNORs), partially retained tetraploidy with secondary diploidization (acipenseriform acrocentric marker, HoxA and HoxD loci) and diploidized characters $\left(\mathrm{DAPI}^{+}\right.$and $\mathrm{CMA}_{3}^{+}$heterochromatic sites). Accordingly, the altogether 120 chromosomes can be arranged into quadruplets, semi-quadruplets and doublets. We suggest that paddlefishes are similar to their relatives, sturgeons, in their propensity for genome duplication and subsequent rediploidization, and that both groups have a good prospect as vertebrate models for further exploration of these processes.

\section{Methods}

Material and metaphase chromosome preparation

Eight individuals of $P$. spathula of unknown sex examined cytogenetically in this study are summarized in the Table 2. They were sacrificed by overdose of anaesthetic 0.5\% Phenoxyethanol (v/v, SIGMA), blood was taken for leucocytes cultivation and fin clips for microsatellite genotyping. Only a fraction of samples yielded metaphase spreads of sufficient quality to be used in molecular cytogenetic analyses.

All fish examined in this study, chromosome preparations, and DNA and tissue samples are deposited in the Laboratory of Fish Genetics, Institute of Animal Physiology and Genetics, Czech Academy of Science (LFG, IAPG, CAS), Liběchov as voucher specimens and reference samples. 
Table $\mathbf{2}$ List of Polyodon spathula specimens analysed cytogenetically

\begin{tabular}{lll}
$\begin{array}{l}\text { Indivuals processed } \\
\text { for chromosomes }\end{array}$ & Origin of specimens & $\begin{array}{l}\text { Individuals used for molecular } \\
\text { cytogenetics (\# metaphases) }\end{array}$ \\
\hline $1-7 / 2008$ & University of South Bohemia in Ceske Budejovice & $1(15) / 2008$ \\
$1-6 / 2009$ & University of South Bohemia in Ceske Budejovice & $1(5), 5(10) / 2009$ \\
$1-8 / 2012$ & Pet shop in Mlada Boleslav, originally from Hungary & $1(2), 4(2), 5(14) / 2012$ \\
$1-8 / 2013$ & Pet shop in Mlada Boleslav, originally from Hungary & $7(11), 8(13) / 2013$ \\
\hline
\end{tabular}

Additionally, fin clips were taken from twenty four individuals originating from a pet shop in 2014 and these were subsequently processed for microsatellite genotyping.

The leucocytes were cultured and chromosome spreads prepared according to the protocol [48] with some modifications described in [13]. To increase the chance of obtaining usable chromosome preparations, some individuals were processed using a direct method according to [13].

\section{Chromosome staining and karyotype analysis}

Paddlefish chromosomes were analysed by Giemsa staining and specific staining and banding methods combined with FISH mapping. Namely, buffered Giemsa ( $\mathrm{pH} 7.0$, $5 \%, 5 \mathrm{~min}$ ) was performed to assess chromosome quality and provide comparison with earlier chromosomal studies. Subsequently, chromosomes were destained by incubation in fixative for $3 \mathrm{~min}$ at RT and briefly washed by distilled water. After air-drying, $\mathrm{CMA}_{3}$ and DAPI fluorescent staining was performed sensu Sola et al. [49]. Finally, the $\mathrm{AgNO}_{3}$ staining sensu Howell and Black [50] and Cbanding sensu Haaf and Schmid [51] were performed. Separately DAPI- and $\mathrm{CMA}_{3}$-stained chromosomes were converted into black and white images, inverted and arranged into karyotypes.

\section{Fluorescence in situ hybridization (FISH) and signal detection}

Whole genomic DNA (gDNA) was isolated from blood, using DNeasy Blood \& Tissue Kit (Qiagen, Hilden, Germany) according to the manufacturer's instructions. The 28S rDNA and 5S rDNA were PCR amplified from gDNA according to [52] and [53]. The amplified fragments were purified using either the QIAquick PCR purification kit (Qiagen) or Qiagen Gel Extraction Kit, according to the manufacturer's instructions. Aliquots of the purified $28 \mathrm{~S}$ rDNA were used in a sequencing reaction using BigDye ${ }^{\oplus}$ Terminator v1.1 Cycle Sequencing Kit (Life Technologies) and subsequently analysed at ABI Prism 3700 Genetic Analyzer. Additionally, the same PCR products were sequenced by the Macrogen Inc. (Seoul, South Korea). Since a preliminary sequence screening has shown an intra-individual sequence variation of the 5SrDNA, purified products from three independent PCR runs were subjected to cloning using the
Qiagen PCR Cloning Kit. The procedure followed the manufacturer's instructions, except for using a half of the recommended amount of the vector and competent cell: $20 \mathrm{ng}$ of the PCR product was ligated to $25 \mathrm{ng}$ of the pDrive cloning vector and a $1 \mu$ l aliquot of the obtained ligation-reaction mixture was used to transform $25 \mu \mathrm{l}$ of Qiagen EZ Competent Cells. These were cultured for $12-24 \mathrm{~h}$ at $37{ }^{\circ} \mathrm{C}$ on LB agar plates containing Ampicillin $(100 \mu \mathrm{g} / \mathrm{ml})$. Randomly selected colonies from each transformed cell lineage were then transferred into the liquid LB medium and cultivated overnight on a shaking platform at $37{ }^{\circ} \mathrm{C}$. Plasmids were isolated and 5SrDNA was extracted using the QIAprep Spin Miniprep Kit (Qiagen). The extract DNA was sequenced in the same way as previously described for $28 \mathrm{~S} \mathrm{rDNA}$.

The nucleotide sequence data have been deposited in GenBank (under accession numbers KM103731KM103735: KM103731 18S rDNA, KM103732 28S rDNA, KM103733- KM103735 5S rDNA).

DNA probes were indirectly labelled with biotin-16dUTP and digoxigenin-11-dUTP (both Roche Diagnostics, Mannheim, Germany) through labelling PCR re-amplification of the previously sequenced PCR products. Reactions were performed in $50 \mu \mathrm{l}$ total volume containing $1 \times$ reaction buffer, $2 \mathrm{mM} \mathrm{MgCl}_{2}$, labelled dNTP nucleotide mix (dATP, dCTP, dGTP each $12.5 \mu \mathrm{M}$, dTTP $8.5 \mu \mathrm{M}$, dUTP conjugated with a hapten - biotin-16-dUTP or digoxygenin-11-dUTP (both Roche), final concentration $4 \mu \mathrm{M}$ ), forward and reverse primer $(0.4 \mu \mathrm{M}$ each), $1.25 \mathrm{U}$ of Taq polymerase (all reagents from Top-Bio, Prague, Czech Republic) and approximately $100 \mathrm{ng}$ of PCR product as template DNA. The dNTP nucleotide mix was prepared as a premix containing $5 \mu \mathrm{dATP}$ dCTP, dGTP (each $2.5 \mathrm{nM}$ ) and $3.4 \mu \mathrm{l}$ dTTP $(2.5 \mathrm{nM}), 4 \mu \mathrm{l}$ dUTP $(1 \mathrm{mM})$ conjugated with a hapten, $27.6 \mu \mathrm{l}$ PCR water in total volume $50 \mu \mathrm{l}$.

FISH with telomeres were performed using the Star*FISH Concentrated Human Chromosome Pan-Telomeric Painting Probe directly labelled with the Cy3 fluorescent dye (Cambio, Cambridge, UK) according to manufacturer's instruction.

The BAC clones of paralogous gene clusters for each of the HoxA and HoxD (Table 1, including accession numbers in GenBank) were produced by Crow et al. 
(2012) [12] and provided for this study. The BAC DNA was labelled indirectly (biotin-16-dUTP and digoxigenin11-dUTP) by nick translation using the Nick Translation Mix (Roche) according to the manufacturer's instructions. The precipitation and resuspension of the probe as well as aging $\left(3-12 \mathrm{~h}\right.$ at $37{ }^{\circ} \mathrm{C}$ and $30 \mathrm{~min}$ at $65^{\circ} \mathrm{C}$ ) and pepsin treatment of the chromosome preparations, hybridization and detection were performed as described in [54]. FISH with the BAC DNA of both paralogs of HoxA and HoxD were performed with and without competitor DNA derived from the Siberian sturgeon (Acipenser baerii) genomic DNA in excess 20-50 times of the probed BAC DNA.

The nomenclature of respective $\operatorname{Hox} A / D \alpha$ and $\beta$ paralogs was adopted from [12]. This nomenclature reflects the fact that these paralogs are products of the paddlefish specific WGD event.

Quantification of repetitive sequences in used BAC clones DNA sequences of BAC clones used as FISH probes originating from [12] (Table 1) have been retrieved from the online "Nucleotide" NCBI database https://www.nc bi.nlm.nih.gov/nuccore according to their GenBank Accession Numbers (Table 1). These sequences have been subjected to screening for interspersed repeats and low complexity DNA sequences with the online tool RepeatMasker [55] using Danio rerio as the DNA source for sequence comparison, 'abblast' search engine, and otherwise default settings. Produced reports are summarized in this study (Table 1) and all complete reports are archived by RS.

\section{Microscopy and image processing}

Chromosome preparations were observed with the AX70 Olympus microscope equipped with a standard fluorescence filter set and captured with a black and white CCD camera separately for each fluorescent dye. Digital images were then pseudo-coloured (blue for DAPI, red for Rhodamine or Cy3, green for Fluorescein or FITC and $\mathrm{CMA}_{3}$ ) and processed in Adobe Photoshop, version CS5. Karyotypes were produced using the IKAROS software (Metasystems, Germany).

\section{Microsatellite analyses}

Twenty four individuals were processed for microsatellite genotyping. The genomic DNA was extracted from fin clips stored in $96 \%$ molecular grade ethanol by the NucleoSpin tissue kit (Macherey-Nagel, Germany) following manufacturer protocol. Nineteen microsatellite markers were tested for amplification using standard gradient - PCR. These markers are listed in Additional file 1 (Supplementary methods).

Markers, which consistently amplified, were selected for subsequent analyses. To avoid fluorescent labeling of each forward primer, forward primers within each of the primer sets possessed a $5^{\prime}$ prime end tail (M13R). During PCR, a fluorescently labelled primer (M13R) was added to the standard amplification reaction [56]. Detailed PCR protocol is listed in Additional file 1 (Supplementary methods). The level of genome reduplication/reduction was investigated as described by [18].

\section{Additional files}

Additional file 1: Supplementary Methods. Microsatellite markers tested for amplification in the present study and PCR protocol. (DOC $45 \mathrm{~kb}$ )

Additional file 2: Supplementary Results. Results of microsatellite genotyping, comparison of number of alleles, allele frequencies by locus. (PDF $83 \mathrm{~kb}$ )

\section{Abbreviations}

BAC (DNA): Bacterial artificial chromosome DNA; CMA3: Chromomycin A3 stain; Cy3: Cytochrome 3; DAPI: 4', 6-diamidino-2-phenylindole stain; FISH: Fluorescence in situ hybridization; FITC: Fluorescein isothiocyanate; gDNA: Genomic DNA; HoxA/D: Homeobox gene clusters A/D; Mya: Millions of years ago; PCR: Polymerase chain reaction; rDNA: Ribosomal deoxyribonucleic acid; WGD: Whole genome duplication

\section{Acknowledgments}

We thank to Marie Rábová for her help during karyotypes preparation and to Jana Čechová, Šárka Pelikánová and Petra Šejnohová for their laboratory assistance.

\section{Funding}

The study was financially supported by the Ministry of Education, Youth and Sports of the Czech Republic projects CENAKVA No. CZ.1.05/2.1.00/01.0024, CENAKVAll No. LO1205 under the NPU I program and by projects of the Czech Science Foundation No.14-28375P and 14-02940S. Further, by the project EXCELLENCE CZ.02.1.01/0.0/0.0/15_003/0000460 OP RDE and with the in stitutional support RVO: 67985904. Granting agencies had no participation in the design of the study or interpretation of the results. This study was also supported by the University of Innsbruck.

\section{Availability of data and materials}

All fish examined in this study, chromosome preparations, and DNA and tissue samples are deposited in the Laboratory of Fish Genetics, Institute of Animal Physiology and Genetics, Czech Academy of Science (LFG, IAPG, CAS), Liběchov as voucher specimens and reference samples.

\section{Authors' contributions}

RS designed the study, prepared the manuscript, performed FISH experiments and the sequence analysis of BAC clones, MH performed the microsatellite analyses and co-drafted the manuscript, CTA provided isolated BACs of Hox paralogs, WMH and PR co-drafted the manuscript, TK took part in the FISH experiments and manuscript preparation, DG and MF provided fish for chromosome preparations, all authors participated on the preparation of the manuscript. All authors read and approved the final manuscript.

\section{Competing interests}

The authors declare that they have no competing interests.

\section{Consent for publication}

Not applicable.

\section{Ethics approval and consent to participate}

All experimental procedures involving fish were approved by the Institutional Animal Care and Use Committee of the IAPG AS CR, according with directives from the State Veterinary Administration of the Czech Republic, permit number 217/2010, and by the permit number CZ 00221 issued by Ministry of Agriculture of the Czech Republic. PR and RS have Certificate of competency according to $\$ 17$ of the Czech Republic Act No. 246/1992 coll. on the 
Protection of Animals against Cruelty (Registration numbers: CZU 955/06 and CZ 00832), provided by Central Commission for Animal Welfare, which authorizes animal experiments in the Czech Republic

\section{Author details \\ 'Laboratory of Fish Genetics, Institute of Animal Physiology and Genetics, Czech Academy of Sciences, 277 21, Liběchov, Czech Republic. ${ }^{2}$ Research Institute for Limnology, University of Innsbruck, Mondseestr. 9, Mondsee, Austria. ${ }^{3}$ University of South Bohemia in České Budějovice, Faculty of Fisheries and Protection of Waters, South Bohemian Research Center of Aquaculture and Biodiversity of Hydrocenoses, 38925 Vodñany, Czech Republic. ${ }^{4}$ Benaroya Research Institute \& University of Washington, Seattle, WA 98101, USA. ${ }^{5}$ Department of Biological and Environmental Sciences, Samford University, 800 Lakeshore Drive, Birmingham, AL 35229, USA.}

Received: 10 September 2016 Accepted: 11 February 2017 Published online: 02 March 2017

\section{References}

1. Nelson JS. Fishes of the world. 4th ed. Hoboken: John Wiley \& Sons; 2006

2. Peng Z, Ludwig A, Wang D, Diogo R, Wei Q, He S. Age and biogeography of major clades in sturgeons and paddlefishes (Pisces: Acipenseriformes). Mol Phylogenet Evol. 2007;42:854-62.

3. Krieger J, Hett AK, Fuerst PA, Artyukhin E, Ludwig A. The molecular phylogeny of the order Acipenseriformes revised. J Appl Ichthyol. 2008;24:36-45.

4. Dingerkus G, Howell WM. Karyotypic analysis and evidence of tetraploidy in the North American paddlefish, Polyodon spathula. Science. 1976;194:842-4.

5. Z Zhang S-M, Yang Y, Deng H, Wei QW, Wu QJ. Genome size and ploidy characters of several species of sturgeons and paddlefishes with comments on cellular evolution of Acipenseriformes. Acta Zool Sinica. 1999;45:200-6.

6. Birstein VJ, Poletaev Al, Goncharov BF. DNA content in Eurasian sturgeon species determined by flow cytometry. Cytometry. 1993;14:377-83.

7. Birstein VJ, Hanner R, Desalle R. Phylogeny of the Acipenseriformes: Cytogenetic and molecular approaches. Environ Biol Fishes. 1997:48:127-56.

8. Billard R, Lecointre G. Biology and conservation of sturgeon and paddlefish. Rev Fish Biol Fisher. 2001;10:355-92.

9. Romanenko SA, Biltueva LS, Serdyukova NA, Kulemzina Al, Beklemisheva VR, Gladkikh OL, et al. Segmental paleotetraploidy revealed in sterlet (Acipenser ruthenus) genome by chromosome painting. Mol Cytogen. 2015;8:90.

10. Havelka M, Kašpar V, Hulák M, Flajšhans M. Sturgeon genetics and cytogenetics: a review related to ploidy levels and interspecific hybridization. Folia Zool. 2011;60:93-103.

11. Trifonov VA, Romanenko SS, Beklemisheva VR, Biltueva LS, Makunin Al, Lemskaya NA, et al. Evolutionary plasticity of acipenseriform genomes. Chromosoma. 2016; doi: 10.1007/s00412-016-0609-2

12. Crow KC, Smith CD, Cheng JF, Wagner GP, Amemiya CA. An independent genome duplication inferred from Hox paralogs in the American paddlefish - a representative basal ray-finned fish and important comparative reference. Genome Biol Evol. 2012:4:937-53.

13. Symonová R, Flajšhans $M$, Sember $A$, Havelka $M$, Gela D, Kořínková $T$, et al. Molecular cytogenetics in artificial hybrid and highly polyploid sturgeons: an evolutionary story narrated by repetitive sequences. Cytogenet Genome Res. 2013;141:153-62.

14. Havelka M, Hulák M, Ráb P, Rábová M, Lieckfeldt D, Ludwig A, et al. Fertility of a spontaneous hexaploid male Siberian sturgeon, Acipenser baerii. BMC Genet. 2014;15:5.

15. Havelka M, Bytyuskyy D, Symonová R, Ráb P, Flajshans M. The second highest chromosome count among vertebrates is observed in cultured sturgeon and is associated with genome plasticity. Genet Sel Evol. 2016:48:12.

16. Wolfe KH. Yesterday's polyploids and the mystery of diploidization. Nat Rev Genet. 2001;2:333-41.

17. Fontana F, Congiu L, Mudrak VA, Quattro JM, Smith TIJ, Ware K, et al. Evidence of hexaploid karyotype in Shortnose sturgeon. Genome. 2008;51:113-9.

18. Havelka M, Hulák M, Bailie DA, Prodöhl PA, Flajšhans M. Extensive genome duplications in sturgeons: new evidence from microsatellite data. J Appl Ichthyol. 2013;29:704-8.

19. Ludwig A, Belfiore NM, Pitra Ch, Svirsky V, Jenneckens I. Genome duplication events and functional reduction of ploidy levels in sturgeon (Acipenser, Huso and Scaphirhynchus). Genetics. 2001;158:1203-15.
20. Macqueen DJ, Johnston IA. A well-constrained estimate for the timing of the salmonid whole genome duplication reveals major decoupling from species diversification. Proc R Soc B. 2014;281:20132881.

21. Vandepoele K, De Vos W, Taylor JS, Meyer A, Van de Peer Y. Major events in the genome evolution of vertebrates: paranome age and size differ considerably between ray-finned fishes and land vertebrates. Proc Natl Acad Sci U S A. 2004; 101:1638-43.

22. McGinnis W, Krumlauf R. Homeobox genes and axial patterning. Cell. 1992; 68:283-302.

23. Garcia-Fernandez J, Holland PWH. Archetypal Organization of the Amphioxus Hox Gene-Cluster. Nature. 1994;370:563-6.

24. Putnam NH, Butts T, Ferrier DEK, Furlong RF, Hellsten U, Kawashima T, et al. The Amphioxus genome and the evolution of the chordate karyotype. Nature. 2008;453:1064-71.

25. Martinez P, Amemiya CT. Genomics of the HOX gene cluster. Comp Biochem Physiol B: Biochem Mol Biol. 2002;133:571-80

26. Van de Peer $Y$, Maere $S$, Meyer $A .2 R$ or not $2 R$ is not the question anymore. Nat Rev Genet. 2010;11:166.

27. May B, Krueger CC, Kincaid HL. Genetic variation at microsatellite loci in sturgeon: primer sequence homology in Acipenser and Scaphirhynchus. Can J Fish Aquat Sci. 1997:54:1542-7.

28. Heist EJ, Nicholson EH, Sipiroski JT, Keeney DB. Microsatellite markers for the American paddlefish (Polyodon spathula). Conserv Genet. 2002;3:205-7.

29. Venkatesh B. Evolution and diversity of fish genomes. Curr Opin Genet Dev. 2003:13:588-92.

30. Arai R. Fish Karyotypes. A Check List. Springer. 2011.

31. Glasauer SM, Neuhauss SC. Whole-genome duplication in teleost fishes and its evolutionary consequences. Mol Genet Genomics. 2014;289:1045-60.

32. Phillips R, Ráb P. Chromosome evolution in the Salmonidae (Pisces): an update. Biol Rev. 2001;76:1-25.

33. Mable BK, Alexandrou MA, Taylor MI. Genome duplication in amphibians and fish: an extended synthesis. J Zool. 2011;284:151-82.

34. Fontana F, Zane L, Pepe A, Congiu L. Polyploidy in Acipenseriformes: cytogenetic and molecular approaches. In: Pisano E, Ozouf-Costaz C, Foresti F, Kapoor BG, editors. Fish Cytogenetics. Enfield: Science Publisher, Inc.; 2007. p. 385-403.

35. Vasilev VP. Mechanisms of polyploid evolution in fish: Polyploidy in Sturgeons. In: Carmona R, Domezain A, García-Gallego M, Hernando JA Rodríguez F, Ruiz-Rejón M, editors. Biology, Conservation and Sustainable Development of Sturgeons. The Netherlands: Springer Science; 2009. p. 97-117.

36. Birstein VJ, Vasil'ev VP. Tetraploid-octoploid relationships and karyological evolution in the order Acipenseriformes (Pisces): karyotypes, nucleoli, and nucleolus-organizer regions in four acipenserid species. Genetica. 1987;72:3-12

37. Krieger J, Fuerst PA. Evidence for a slowed rate of molecular evolution in the order Acipenseriformes. Mol Biol Evol. 2002;19:891-7.

38. Ráb P. Karyotype evolution in fishes of the order Esociformes. Habilitation Thesis. Praque: Charles University in Prague (in Czech); 2004.

39. Stimpson KM, Sullivan LL, Kuo ME, Sullivan BA. Nucleolar organization, ribosomal DNA array stability, and acrocentric chromosome integrity are linked to telomere function. PLoS ONE. 2014;9(3), e92432.

40. Symonová R, Majtánová Z, Korínková T, Jankun M, Dion-C té A-M, Bernatchez L, Ráb P. Chromosomal characteristics of rDNA genes in salmonid fishes: trends in their patterns and evolution. 20th International Colloquium on Animal Cytogenetics and Gene Mapping, Cordoba, Spain. Chrom Res. 2012;20(6):810.

41. Symonová R, Majtánová Z, Sember A, Staaks GBO, Bohlen J, Freyhof J, et al. Genome differentiation in a species pair of coregonine fishes: an extremely rapid speciation driven by stress-activated retrotransposons mediating extensive ribosomal DNA multiplications. BMC Evol Biol. 2013;13:42.

42. Sember A, Bohlen J, Šlechtová V, Symonová R, Ráb P. Karyotype differentiation in nemacheilid loach fishes (Nemacheilidae, Cobitoidea, Cypriniformes): hidden variability uncovered by molecular cytogenetic markers in 19 species and its phylogenetic interpretation. BMC Evol Biol. 2015:15:251

43. David L, Blum S, Feldman MW, Lavi U, Hillel J. Recent duplication of the common carp (Cyprinus carpio L.) genome as revealed by analyses of microsatellite loci. Mol Biol Evol. 2003;20:1425-34.

44. Knytl M, Kalous L, Symonová R, Rylková K, Ráb P. Chromosome studies of European cyprinid fishes: cross-species painting reveals natural allotetraploid origin of a Carassius female with 206 chromosomes. Cytogenet Genome Res. 2013;139:276-83. 
45. Minguillón C, Gardenyes J, Serra E, Castro LF, Hill-Force A, Holland PW, et al. No more than 14: the end of the amphioxus Hox cluster. Int J Biol Sci. 2005; 1:19-23.

46. Birstein VJ. Phylogeny and evolution of Acipenseriformes: new molecular and genetic data create new puzzles. In: Gall YM, Kolchinsky El, editors. Evolutionary biology: history and theory, vol. 3. St. Peterburg: Nauka; 2005. p. 231-69.

47. Allendorf FW, Thorgaard GH. Tetraploidy and the evolution of salmonid fishes. In: Turner BJ, editor. Evolutionary Genetics of Fishes. New York: Plenum Press; 1984. p. 1-46.

48. Fujiwara A, Hishida-Umehara C, Sakamoto T, Okamoto N, Nakyama I, Abe S. Improved fish lymphocyte culture for chromosome preparation. Genetica. 2001:111:77-89.

49. Sola L, Rossi AR, laselli V, Rasch EM, Monaco PJ. Cytogenetics of bisexual/ unisexual species of Poecilia. II. Analysis of heterochromatin and nucleolar organizer regions in Poecilia mexicana mexicana by C-banding and DAPI, quinacrine, chromomycin A3 and silver staining. Cytogenet Cell Genet. 1992;60:229-35.

50. Howell WM, Black DA. Controlled silver-staining of nucleolus organizer regions with a protective colloidal developer: A 1-step method. Experientia. 1980;36:1014-5.

51. Haaf T, Schmid M. An early stage of ZW/ZZ sex chromosome differentiation in Poecilia sphenops var. melanistica (Poeciliidae, Cyprinodontiformes). Chromosoma. 1984;89:37-41.

52. Dayrat B, Tillier A, Lecointre G, Tillier S. New clades of euthyneuran gastropods (Mollusca) from 28S rRNA sequences. Mol Phylogenet Evol. 2001;19:225-35.

53. Komiya $\mathrm{H}$, Takemura S. Nucleotide sequence of $5 \mathrm{~S}$ ribosomal RNA from rainbow trout (Salmo gairdnerii) liver. J Biochem. 1979;86:1067-80.

54. Symonová R, Sember A, Majtánová Z, Ráb P. Characteriazation of Fish Genomes by GISH and CGH. In: Ozouf-Costaz C, Pisano E, Foresti F, De Almeida F, Toledo L, editors. Fish Cytogenetic Techniques. Ray-Fin Fishes and Chrondrichthyans. CRC Press. 2015. p. 118-31.

55. Smit AFA, Hubley R, Green P. Repeat Masker Open-3.0. 2010. www. repeatmasker.org

56. Schuelke M. An economic method for the fluorescent labeling of PCR fragments: a poor man's approach to genotyping for research and highthroughput diagnostics. Nat Biotechnol. 2000;18:233-4.

\section{Submit your next manuscript to BioMed Central and we will help you at every step:}

- We accept pre-submission inquiries

- Our selector tool helps you to find the most relevant journal

- We provide round the clock customer support

- Convenient online submission

- Thorough peer review

- Inclusion in PubMed and all major indexing services

- Maximum visibility for your research

Submit your manuscript at www.biomedcentral.com/submit

) Biomed Central 